\title{
Kidney disease in the Global Burden of Disease Study 2017
}

Simon DS Fraser ${ }^{1 *}$ and Paul J Roderick ${ }^{1 *}$

${ }^{1}$ School of Primary Care and Population Sciences, Faculty of Medicine, University of Southampton, Southampton, UK

* email: s.fraser@soton.ac.uk, ORCID 0000-0002-4172-4406; pjr@soton.ac.uk

Refers to GBD 2017 Disease and Injury Incidence and Prevalence Collaborators. Global, regional, and national incidence, prevalence, and years lived with disability for 354 diseases and injuries for 195 countries and territories, 1990-2017: a systematic analysis for the Global Burden of Disease Study 2017. Lancet. 392, 1789-1858 (2018).| GBD 2017 DALYs and HALE Collaborators. Global, regional, and national disabilityadjusted life-years (DALYs) for 359 diseases and injuries and healthy life expectancy (HALE) for 195 countries and territories, 1990-2017: a systematic analysis for the Global Burden of Disease Study 2017. Lancet. 392, 1859-1922 (2018).| GBD 2017 Risk Factor Collaborators. Global, regional, and national comparative risk assessment of 84 behavioural, environmental and occupational, and metabolic risks or clusters of risks for 195 countries and territories, 1990-2017: a systematic analysis for the Global Burden of Disease Study 2017. Lancet. 392, 1923-1994 (2018).

The analyses in the 2017 Global Burden of Disease Study demonstrate the growing burden of chronic kidney disease (CKD), mainly driven by population ageing; absolute levels for every CKD metric considered rose significantly, whereas age-standardised rates were fairly stable. The prevalence of key metabolic CKD risk factors, particularly obesity, also show a worrying increase.

The 2017 Global Burden of Disease (GBD) Study captured and summarised data from 1990 to 2016 on death and disability for over 350 diseases and injuries across 195 countries; it also, included the analysis of 84 risk factors for which evidence of a causal risk-condition relationship exists. The study includes analysis of chronic kidney disease (CKD; based on the international classification of diseases (ICD) coding of hospitalisation and mortality) and of impaired kidney function (IKF; defined as the proportion of the population with albumin to creatinine ratio $(A C R)>30 \mathrm{mg} / \mathrm{g}$ or estimated glomerular filtration rate $\left(\right.$ eGFR) $<60 \mathrm{~mL} / \mathrm{min} / 1 \cdot 73 \mathrm{~m}^{2}$, excluding end-stage renal disease) as a risk factor for key non-communicable diseases (NCDs), notably cardiovascular disease and CKD. Analyses of the main burdens attributed to CKD and IKF in 2007-2017 show a significant rise of at least $20 \%$ in absolute levels for every CKD metric (TABLE 1$)^{1-4}$. However, CKD age-standardised rates, which adjust for population growth and ageing, were generally stable. Using absolute counts, CKD ranks in the top 30 of all diseases for several metrics and moved up in ranking for mortality and disability adjusted life years (DALYs, a composite of years of life lost (YLL, a metric of mortality) and years lived with disability $(\mathrm{YLD}))^{3}$. Of note, global absolute CKD prevalence increased by $28.2 \%$ from $2007-2017$ among females and $25.4 \%$ among males, whereas the increases in age-standardised rates were $3.0 \%$ and $1.1 \%$ respectively ${ }^{1}$. The 
age-standardised prevalence of IKF was also presented as the summary exposure value (SEV), which was stable for males but increased slightly $(1.7 \%)$ in females ${ }^{4}$.

Arguably, one of the most important measures presented is the DALY, which allows comparison with other disease states and incorporates both death and disability but requires assumptions to be made about disease severity and disability weights. Both the CKD burden by absolute count and by all-age DALY rate, which adjusts for population growth, are increasing globally and this is reflected in the contribution of IKF to the NCD DALY burden ${ }^{3,4}$. Age-standardised CKD DALY rates have not increased. CKD due to type 2 diabetes mellitus (T2DM) represented the second largest increase in DALYs for all conditions analysed (34.3\%) between 2007 and $2017^{3}$.

A forecasting analysis suggests that the number of global YLL due to CKD will increase from $~ 26$ million in 2016 to 52.5 million in 2040, and deaths will rise from 1.2 million in 2016 to 3.1 million in $2040^{5}$. These increases are predicted to move CKD in the YLL rankings from $16^{\text {th }}$ in 2016 to $5^{\text {th }}$ in 2040 , a change thought to be mainly driven by population growth and ageing, although the modelling assumptions are complex ${ }^{5}$. It is possible that mortality or YLL attributable to CKD may be underestimated in these analyses, given the underuse of CKD codes on death certification, which has implications for the reliability of modelling future YLL trajectories.

One GBD study analysed four key CKD risk factors: dietary sodium, fasting glucose, body mass index (BMI) and systolic blood pressure (SBP) ${ }^{4}$. The last three of these metabolic risk factors were in the top five of absolute contributions to all DALYs ${ }^{4}$. Between 1990 and 2017, overall SEV of BMI increased by $70 \%$ and that of high fasting glucose by $38 \%$, whereas the overall SEV of high SBP decreased by $2 \%$. In absolute terms, the CKD DALYs attributed to high sodium, high fasting glucose, high BMI and high SBP were 3,400,000, $20,620,000,9,530,000$ and 17,490,000, respectively. There were considerable absolute percentage increases for all of these CKD risk factors since 2007 - highest for BMI at over $40 \%$ - but percentage changes in agestandardised rates were much smaller, generally $<5 \%$, except for $\mathrm{BMI}^{4}$.

Certain regions are experiencing a dramatic increase in CKD burden ${ }^{6}$, including Oceania, South Asia, East Asia, Caribbean, Central and Tropical Latin America, North Africa and the Middle East ${ }^{1}$. In terms of all-age DALY rate, Central Latin America shows the most concerning increase and this trend is only partly attenuated by age-standardisation. ${ }^{3}$ Death rates attributed to CKD show a similar pattern in these regions, as does the contribution of IKF as an NCD risk factor ${ }^{4}$. Middle socio-demographic index (SDI) regions are experiencing greater increases in deaths attributed to CKD and in the importance of IKF as a risk factor for NCDs than those with low or high SDI. Of note, whilst global GBD analyses break down by gender, socio-economic factors are only examined at country level using SDI, and there is no analysis by major ethnic groups. Similar increases are seen for CKD related to T2DM, particularly in South and East Asia, Central Latin America, North Africa and the Middle East. ${ }^{1,3}$. 
The GBD study has important limitations. Methods for generating the diverse metrics globally and by time period, country, region and person are complex and difficult to fully appraise. Moreover, the sources of data used by GBD analyses are increasing rapidly but considerable assumptions are needed, especially for countries where data are sparse. The data representativeness index (that is, the percentage of locations with data for a given time period) for IKF in 2007-2017 was only $28.5 \%{ }^{5}$. In addition, estimating the impact of CKD as a condition depends on the validity of ICD coding, particularly when categorised into disease subtypes, and health survey data, which is widely used for CKD prevalence estimation, are largely based on eGFR and ACR without specifying underlying cause ${ }^{7}$. In Europe, substantial inter-country variation in CKD prevalence was not explained by the prevalence of diabetes, hypertension or obesity ${ }^{7}$. This variation is more likely related to social, environmental and genetic factors than to survey and laboratory assay issues, but such risks are not explicitly captured in the GBD study.

Despite some uncertainties in the data assumptions, the latest GBD study highlights the growing absolute burden of CKD across all regions, largely driven by population ageing, and a global rise in metabolic risk factors (particularly obesity), especially in Latin America and Caribbean, North Africa and Middle East, and Oceania. These trends dictate that policies and practices should focus on increasing the effectiveness of CKD prevention strategies by tackling the rise in obesity and high fasting glucose. Improvements are required in identifying and managing individuals at high risk of CKD (such as those with hypertension or diabetes) to aid disease prevention, and in the management of patients with CKD to help to minimise the risk of CKD progression and of cardiovascular disease. The rising prevalence of CKD, which usually co-exists with other morbidities, will add to the complexity of patient management and lead to a higher treatment burden for patients, as well as a greater need for prevention and management of CKD-associated acute kidney injury ${ }^{8}$. Equitable and effective delivery of renal replacement therapy will be essential to tackle the inevitable rise in the incidence of ESRD, which will have a substantial cost impact for health systems, especially in low and middleincome countries ${ }^{9}$. The need for kidney transplantation will increase and ensuring an adequate, skilled nephrology workforce will be a challenge. Finally, prevention and management of CKD requires a wholesystem approach, including universal health coverage and adequately-funded and co-ordinated primary, secondary and tertiary services, to address the considerable variation in the capacity for kidney care between and within regions, as highlighted by the Global Kidney Health Care Status survey ${ }^{10}$.

\section{References}

1. GBD 2017 Disease and Injury Incidence and Prevalence Collaborators. Global, regional, and national incidence, prevalence, and years lived with disability for 354 diseases and injuries for 195 countries and territories, 1990-2017: a systematic analysis for the Global Burden of Disease Study 2017. Lancet. 392, 1789-1858 (2018). 
2. GBD 2017 Causes of Death Collaborators. Global, regional, and national age-sex-specific mortality for 282 causes of death in 195 countries and territories, 1980-2017: a systematic analysis for the Global Burden of Disease Study 2017. Lancet. 392,1736-1788 (2018).

3. GBD 2017 DALYs and HALE Collaborators. Global, regional, and national disability-adjusted life-years (DALYs) for 359 diseases and injuries and healthy life expectancy (HALE) for 195 countries and territories, 1990-2017: a systematic analysis for the Global Burden of Disease Study 2017. Lancet. 392 , 1859-1922 (2018)

4. GBD 2017 Risk Factor Collaborators. Global, regional, and national comparative risk assessment of 84 behavioural, environmental and occupational, and metabolic risks or clusters of risks for 195 countries and territories, 1990-2017: a systematic analysis for the Global Burden of Disease Study 2017. Lancet. 392, 1923-1994 (2018).

5. Foreman KJ et al. Forecasting life expectancy, years of life lost, and all-cause and cause-specific mortality for 250 causes of death: reference and alternative scenarios for 2016-40 for 195 countries and territories. Lancet. 392, 2052-2090 (2018).

6. Jager KJ, Fraser SDS. The ascending rank of CKD in the Global Burden of Disease study. Nephrol Dial Transplant; 32(suppl_2), ii121-ii128 (2017). 9

7. Brück $\mathrm{K}$ et al. CKD prevalence varies across the European general population. J Am Soc Nephrol. 27, 2135-2147 (2016).

8. Tonelli $\mathrm{M}$ et al. Comparison of the complexity of patients seen by different medical subspecialists in a universal health care system. JAMA Network Open 1, e184852. (2018)

9. Pecoits-Filho R, Sola L, Correa-Rotter R et al. Kidney disease in Latin America: current status, challenges and the role of ISN in the development of nephrology in the region. Kidney International 94:1069-72 (2018).

10. Bello AK et al. Assessment of global kidney health care status. JAMA 317, 864-81. (2017) 


\section{Competing interests}

The authors declare no competing interests.

Table 1/ Global metrics for CKD as a condition and impaired kidney function as a risk factor

\begin{tabular}{|c|c|c|c|c|c|c|c|c|c|}
\hline & \multicolumn{7}{|c|}{ CKD } & \multicolumn{2}{|c|}{$\begin{array}{l}\text { (KF as a risk factor } \\
\text { for NCDs }\end{array}$} \\
\hline & \multicolumn{2}{|c|}{$\begin{array}{l}\text { Number of cases } \times 1,000(95 \% \\
\mathrm{Cl})\end{array}$} & \multirow{2}{*}{$\begin{array}{l}\text { Change in } \\
\text { number of } \\
\text { cases } \\
2007-2017 \\
(\%)\end{array}$} & \multirow{2}{*}{$\begin{array}{l}\text { Age- } \\
\text { standardised } \\
\text { rate in } 2017 \\
\text { per } 100,000 \\
(95 \% \mathrm{Cl})\end{array}$} & \multirow{2}{*}{$\begin{array}{l}\text { Change in } \\
\text { age- } \\
\text { standardised } \\
\text { rate } 2007 \text { - } \\
2017(\%)\end{array}$} & \multicolumn{2}{|l|}{ Rank $^{*}$} & \multirow{2}{*}{$\begin{array}{l}\text { Change in } \\
\text { count 2007- } \\
2017(\%)\end{array}$} & \multirow{2}{*}{$\begin{array}{l}\text { Change in } \\
\text { age- } \\
\text { standardised } \\
\text { rate } 2007 \text { - } \\
2017(\%)\end{array}$} \\
\hline & 2007 & 2017 & & & & 2007 & 2017 & & \\
\hline Incidence & $\begin{array}{l}15,001 \\
(13,616 \text { to } 16,358)\end{array}$ & $\begin{array}{l}19,736 \\
(17,727 \text { to } \\
21,983)\end{array}$ & $+31.6 \%$ & $\begin{array}{l}248.0 \\
(223.9 \text { to } 275.8)\end{array}$ & $+4.2 \%$ & $\begin{array}{l}\text { Not in } \\
\text { top } 30\end{array}$ & $\begin{array}{l}\text { Not in top } \\
30\end{array}$ & NA & NA \\
\hline Prevalence & $\begin{array}{l}549,206 \\
(512,807 \text { to } \\
588,533)\end{array}$ & $\begin{array}{l}697,510 \\
(649,209 \text { to } \\
752,051)\end{array}$ & $+27.0 \%^{\$}$ & $\begin{array}{l}8724.1 \\
(8124.3 \text { to } \\
9403.5)\end{array}$ & $+2.2 \%$ & 15 & 15 & $\begin{array}{c}+9.9 \% \\
\text { (Exposure - } \\
\text { all age rate } \\
\text { per } 100)\end{array}$ & $\begin{array}{l}+1.1 \% \\
\text { (Exposure - } \\
\text { increase in per } \\
100)\end{array}$ \\
\hline Mortality & $\begin{array}{l}920 \\
\text { (899 to } 954)\end{array}$ & $\begin{array}{l}1230 \\
(1195 \text { to } \\
1259)\end{array}$ & $+34.0 \%$ & $\begin{array}{l}15 \cdot 9 \\
(15 \cdot 5 \text { to } 16 \cdot 3)\end{array}$ & $+1 \cdot 5 \%$ & 14 & 12 & $+26.6 \%$ & $-5.8 \%$ \\
\hline YLL & $\begin{array}{l}23,551 \\
(22,923 \text { to } 24,453)\end{array}$ & $\begin{array}{l}28,508 \\
(27610 \text { to } \\
29314)\end{array}$ & $+21.0 \%$ & $\begin{array}{l}359.4 \\
(348.2 \text { to } 369.6)\end{array}$ & $-2.5 \%$ & 16 & 16 & $+19.0 \%$ & $-6.6 \%$ \\
\hline YLD & $\begin{array}{l}5874 \\
(4384 \text { to } 7393)\end{array}$ & $\begin{array}{l}7306 \\
(5435 \text { to } \\
9215)\end{array}$ & $+24 \cdot 4 \%^{\$}$ & $\begin{array}{l}91.9 \\
(68.2 \text { to } 115.7)\end{array}$ & $-0.2 \%$ & $\begin{array}{l}\text { Not in } \\
\text { top } 30\end{array}$ & $\begin{array}{l}\text { Not in top } \\
30\end{array}$ & $+28.0 \%$ & $+1.6 \%$ \\
\hline DALY & $\begin{array}{l}29,425 \\
(27,742 \text { to } 31,213)\end{array}$ & $\begin{array}{l}35,815 \\
(33,728 \text { to } \\
37,983)\end{array}$ & $+21 \cdot 7 \%^{\$}$ & $\begin{array}{l}451 \cdot 3 \\
(424 \cdot 9 \text { to } \\
478 \cdot 3)\end{array}$ & $-2 \cdot 1 \%$ & 21 & 19 & $+20.3 \%$ & $-5.5 \%$ \\
\hline
\end{tabular}

\#Data are taken from the Lancet Global Burden of Disease (GBD) publications and from the GBD data visualisation hub (https://vizhub.healthdata.org/gbd-compare/) which provides additional information. * based on count among all top 30 conditions.

${ }^{\$}$ Statistically significant change

CKD, chronic kidney disease; DALY, disability-adjusted life years; IKF, impaired kidney function; NCDs, non-communicable diseases;

YLD, years lived with disability; YLL, years life lost. 